\title{
Comparison of systemic effects of midazolam, ketamine, and isoflurane anaesthesia in rabbits
}

\author{
Güneri Atalan ${ }^{1}$, Gültekin Atalan², Hanifi Erol', \\ Muharrem Erol ${ }^{3}$, Ayhan Atasever ${ }^{4}$, Zafer Doğan ${ }^{5}$, \\ Vehbi Güneş $^{6}$, M. Kaan Yönez ${ }^{2}$, Ihsan Keleş ${ }^{6}$

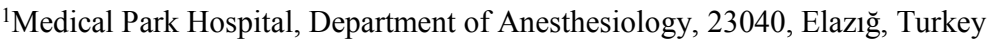 \\ ${ }^{2}$ Department of Surgery, ${ }^{4}$ Department of Pathology, ${ }^{6}$ Department of Internal Medicine, \\ Veterinary Faculty, Erciyes University, 38250, Kayseri, Turkey \\ ${ }^{3}$ Department of Surgery, Veterinary Faculty, Balıkesir University, 10100, Balıkesir, Turkey \\ ${ }^{5}$ Veterinary Surgeon, 34149, Istanbul, Turkey \\ drhaneroll@yahoo.com
}

Received: December 18, $2018 \quad$ Accepted: April 16, 2019

\begin{abstract}
Introduction: Clinical doses of anaesthetic agents were administered to rabbits and effects on the brain, heart, and liver were investigated biochemically and histopathologically. Material and Methods: The rabbits were randomly divided into three main groups (16 rabbits each) and each group into study $(n=8)$ and control $(n=8)$ groups. All study group rabbits received $3 \mathrm{mg} / \mathrm{kg}$ of midazolam (M) intramuscularly. Group 1.1 (M) received nothing further, group 2.1 (MK) also received $25 \mathrm{mg} / \mathrm{kg}$ of ketamine, and group 3.1 (MKI) besides ketamine was also given 2\% isoflurane to induce anaesthesia for $30 \mathrm{~min}$. $\mathrm{NaCl}$ solution in the same volume as midazolam and ketamine was injected into the controls. Results: In clinical evaluation significant differences were detected in respiratory and heart rates. In blood gas analysis the $\mathrm{PO}_{2}$ and $\mathrm{PCO}_{2}$ values showed statistical differences in anaesthesia intervals. Significant biochemical value changes were recorded in creatine kinase-Mb, glucose, and total protein. Histopathological liver examinations revealed higher total apoptotic and normal cell numbers in the MK than in the M and MKI groups. Apoptotic cell numbers were statistically significant in M and MK groups. Conclusion: Anaesthetic agents may increase programmed apoptosis. The MKI anaesthetics combination was found to cause less cell destruction in general than the other study groups. It was indicated that MKI was the safer anaesthetic combination in rabbits.
\end{abstract}

Keywords: isoflurane, ketamine, midazolam, systemic effects, histopathology.

\section{Introduction}

The anaesthetic agents (xylazine, midazolam, detomidine, and ketamine) have adverse side effects on the cardiorespiratory, cardiovascular, and nervous systems. It is desirable that ideal anaesthesia protocols have the least harmful effects on these systems and their organs and are as safe as possible. Therefore, studies investigating the toxic effects of different anaesthetics or their combinations on body systems do not abate.

The most commonly used anaesthetic agents in veterinary medicine are midazolam, ketamine, and isoflurane. However, side effects of these drugs such as hypotension, bradycardia, and apnea, and some toxic effects in tissue have been reported $(14,16,17)$.

Benzodiazepines such as midazolam are potentially sedative-hypnotic and are used as injectable anaesthetics. When used in clinical doses, respiratory and heart rates, tidal volume, blood $\mathrm{pH}$, blood gases, cardiac output, and mean arterial blood pressure do not change $(6,19,22)$.

On the other hand, some harmful effects in individuals with liver and kidney diseases have been proven and reported to accumulate in the body with repeated doses (6). Neurotoxic effects of midazolam have been determined. Furthermore, vascular lesions have been shown in in vivo studies. It has also been 
reported that apoptosis by anaesthetics at high doses may lead to necrosis in various tissues $(7,12,26)$.

Ketamine is one of the most commonly used anaesthetic agents in experimental animals (19). Contrary to most anaesthetic agents, it does not reduce heart rate nor cause myocardial depression (9). Inhaled anaesthetic agents are used for both safe induction and continuation of anaesthesia. At higher concentrations than the minimum alveolar concentration, isoflurane increases intracranial pressure, affects liver functions minimally, and reduces kidney blood flow. However, it does not result in functional or pathological renal impairment due to the minimum level of inorganic fluorine and urea exchange considered harmful to the organism being higher than that caused by isoflurane $(11,14)$.

In recent years it has been shown that anaesthetic agents may induce apoptosis at different doses $(7,12$, 26). However, studies are still underway to determine whether the agents induce apoptosis precisely, and it has also been reported that the rate of apoptosis is higher in those who experience more potent effects than others from the same volume dose of anaesthetic agent. Studies have also reported that apoptosis caused by a combination of synergistic anaesthetic agents is higher than that caused by single agents (21). As a corroborative example, Braun et al. (5) investigated apoptosis induced by ketamine in human cells and reported that ketamine leads to apoptosis in neural and non-neural cells even at millimolar doses. In contrast, Fredrikson et al. (13) reported that ketamine, an NMDA receptor antagonist, does not cause apoptosis, even in neonatal brain cells.

In the present study, the application of the anaesthesia protocol to rabbits at normal clinical doses was to investigate whether they cause apoptosis, especially in the brain, heart, and liver. Additionally, other harmful effects were also investigated and their possible effects were substantiated by biochemical parameters and histopathological studies. All the results were reflected in the field of veterinary anaesthesiology.

\section{Material and Methods}

Animals. A total of 48 New Zealand rabbits (24 female and 24 male), four months old and weighing $2.24 \pm 0.57 \mathrm{~kg}$ were used. Each of the rabbits was kept in a separate cage in the Erciyes University, Experimental Research, Application, and Research Center until the study began. Ad libitum feeding was allowed. The average room temperature and lighting were set at $21 \pm 1^{\circ} \mathrm{C}$ and $12 \mathrm{~h}$ light and $12 \mathrm{~h}$ dark. The rabbits were randomly divided into three main groups and each had 16 rabbits. Each group was also divided into two subgroups: a study $(\mathrm{n}=8)$ and a control $(n=8)$.
Study groups and anaesthesia protocol. Anaesthetic-differentiated study groups were designed as below:

Group $1.1(\mathrm{n}=8)$ : midazolam group $(\mathrm{M})$ administered $3 \mathrm{mg} / \mathrm{kg}$ of midazolam intramuscularly (IM);

Group $1.2(\mathrm{n}=8)$ : midazolam control $(\mathrm{MC})$ administered $\mathrm{NaCl}$ solution equal to midazolam volume IM;

Group $2.1(\mathrm{n}=8)$ : midazolam-ketamine (MK) administered $3 \mathrm{mg} / \mathrm{kg}$ of midazolam and $25 \mathrm{mg} / \mathrm{kg}$ of ketamine (10 min after midazolam administration) IM;

Group $2.2(n=8)$ : midazolam-ketamine control (MKC) administered $\mathrm{NaCl}$ solution equal to volume of midazolam and ketamine IM;

Group $3.1(n=8)$ : midazolam-ketamine-isoflurane (MKI) administered $3 \mathrm{mg} / \mathrm{kg}$ of midazolam and $25 \mathrm{mg} / \mathrm{kg}$ of ketamine (10 min later) IM, intubated $10 \mathrm{~min}$ later and maintained under anaesthesia for 30 min with $2 \%$ isoflurane and $1 \% \mathrm{O}_{2}$;

Group $3.2(n=8)$ : midazolam-ketamine-isoflurane control (MKIC): administered $\mathrm{NaCl}$ solution IM twice in the same volume of midazolam and ketamine as in the group 3.1 .

The intubation of group 3.1 was through $2.5 \mathrm{~mm}$ cuffed tube and was achieved by visualising the tracheal passage with the aid of a laryngoscope (Üzümcü, Turkey). Anaesthesia was carried out with a Classic 2000 anaesthesia machine (SMS Medical Company, Turkey).

Sampling periods and analysis. A blood sample $(1.5 \mathrm{~mL})$ was drained from the vena auricularis for blood gas and serum biochemical analysis before anaesthesia (0 min) and at 10, 30, 60, 180, and $300 \mathrm{~min}$ after midazolam and midazolam-ketamine administration, and isoflurane anaesthesia. Each rabbit was decapitated at $300 \mathrm{~min}$ of anaesthesia.

Blood LDH samples were centrifuged for $15 \mathrm{~min}$ at 3,000 rpm in a Rotafix 32A machine (Hettich, USA), and serum samples were obtained. They were stored at $-20{ }^{\circ} \mathrm{C}$ until analysis. Biochemical analysis of serum was carried out in a fully automatic Cobas Integra auto analyser (Roche Diagnostics GmbH, Germany). The values of alanine aminotransferase (ALT), aspartate aminotransferase (AST), lactate dehydrogenase (LDH), alkaline phosphatase (ALP), and creatine kinase-Mb $(\mathrm{Ck}-\mathrm{Mb})$ activities, albumin, total protein (TP), creatine $(\mathrm{Cr})$, blood urea nitrogen (BUN), cardiac troponin I (cTnI), and glucose (Glu) levels were measured with a Cobas E 411 analyser (Roche Diagnostics GmbH, Germany) using their commercial kits.

Venous blood gas analyses. Samples were analysed by $\mathrm{CC}$ cartridge in an IRMA True (LifeHealth, USA) device. Measurement of $\mathrm{pH}, \mathrm{PCO}_{2}$ $\mathrm{mmHg}, \mathrm{PO}_{2} \mathrm{mmHg}, \mathrm{Htc} \%, \mathrm{Na} \mathrm{mM} / \mathrm{L}, \mathrm{K} \mathrm{mM} / \mathrm{L}$, $\mathrm{Ca} \mathrm{mM} / \mathrm{L}, \mathrm{HCO}_{3} \mathrm{meq} / \mathrm{L}, \mathrm{tCO}_{2} \mathrm{mM} / \mathrm{L}, \mathrm{BE} \mathrm{mM} / \mathrm{L}$, BEecf $\mathrm{mM} / \mathrm{L}, \mathrm{O}_{2} \mathrm{SAT} \%$, and total $\mathrm{Hb}(\mathrm{tHb})$ values were determined before anaesthesia and during 
midazolam, midazolam-ketamine, and isoflurane anaesthesia intervals for the control and study groups.

Clinical evaluations. Heart (HR) and respiratory rates (RR), body temperature (BT), anaesthesia depth, and encountered complications were recorded before and during anaesthesia intervals for the control and study groups. Hypothermia, bradycardia, and hypoventilation were monitored during anaesthesia.

Histopathological analyses. A systemic necropsy was performed on each rabbit (8). Tissue samples of the liver, heart, brain, lungs, spleen, stomach, intestine, pancreas, and adrenal gland were fixed in $10 \%$ formalin. Paraffin sections of $5 \mu \mathrm{m}$ thickness were stained with haematoxylin and examined under a BX51 light microscope (Olympus, Japan). Some sections from the liver, heart muscle, and brain were prepared on adhesive slides in order to determine apoptotic and necrotised cells. Terminal deoxynucleotidyl transferase dUTP nick end labelling (TUNEL) was used to derive the apoptotic index in the form of an In Situ Cell Death Detection Kit, POD (Roche Diagnostics, Germany).

Statistical analysis. Comparisons within and between groups were made using SPSS software version 22 in Windows 7 (IBM, USA). Arithmetic mean $(\mathrm{M}) \pm$ standard deviation (SD) values of the data were considered. Friedman analysis and the KruskalWallis test were used for the repeated measurement, and a chi-square test was carried out for histopathological findings. $\mathrm{P}<0.05$ and $\mathrm{P}<0.001$ were considered significant. Control group values were similar to each other and were also not statistically significant. The mean values of all control groups (MC, $\mathrm{MKC}$, and MKIC values) were used as one set of control group values (Tables 1, 2, 3, and 4).

\section{Results}

Clinical findings. BT had decreased significantly at all sampling times compared to 0 time in all study groups (Table 1). There were significant differences in RR between the baseline ( 0 time) and 10, 30, 60, and $180 \mathrm{~min}$ points in all study groups. RR decreased starting from $10 \mathrm{~min}$ after anaesthesia and continuing to $180 \mathrm{~min}$, although it tended to increase at $300 \mathrm{~min}$ after anaesthesia. A significant difference was determined in HR between the baseline ( 0 time) and 10,30, and $60 \mathrm{~min}$ points after anaesthesia in the MK group. Furthermore, a significant difference was also recorded between the baseline value and values at 10 and $30 \mathrm{~min}$ after anaesthesia in the MKI group. The lowest and highest values of HR were at 30 and $300 \mathrm{~min}$, respectively, in the MKI group.

Venous blood gas analyses. When venous blood $\mathrm{pH}$ values were compared within groups, there was a significant difference between the baseline and 10 , $30,60,180$, and 300 min points after anaesthesia in the MKI group (Table 2). Significant differences in $\mathrm{pH}$ were also found at 10, 30, 60, and $180 \mathrm{~min}$ after anaesthesia when a comparison was made between the control and study groups. Differences were also recorded of $\mathrm{PCO}_{2}$ in the $\mathrm{M}, \mathrm{MK}$, and $\mathrm{MKI}$ groups between the baseline and anaesthesia intervals. These differences were significant between control and study groups. Statistical difference in relation to $\mathrm{PO}_{2}$ was significant between the baseline and $180 \mathrm{~min}$ point in the $\mathrm{M}$ group, between the baseline and 30,180, and 300 min points in the MK group, and between the baseline and 60, 180, and 300 min points in the MKI group. $\mathrm{PO}_{2}$ values were also statistically different when study and control groups were compared. Differences were recorded in $\mathrm{HCO}_{3}, \mathrm{tCO}_{2}, \mathrm{BE}$, BEecf, and $\mathrm{O}_{2} \mathrm{SAT}$ values in study and control groups. Statistically significant differences of $\mathrm{HCO}_{3}$ were detected at 0,60 , and $180 \mathrm{~min}$ of anaesthesia in the MKI group. The MK group tCO2 values disclosed significant differences at 0,30 , and $60 \mathrm{~min}$, and the MKI group did at 0, 10, and $30 \mathrm{~min}$. The increases and decreases of BE, BEecf, and $\mathrm{O}_{2} \mathrm{SAT}$ values were in reference ranges in study and control groups.

Biochemical findings. The results of biochemical analysis in all groups are given in Table 3. AST values at 30 and 60 min were higher in study groups than control groups but they were in reference ranges. The difference in BUN value in the $\mathrm{M}$ group was significant between the baseline and 10, 60, and 180 min points after anaesthesia. Some variations in BUN values were also determined for the other groups but they were within reference values. There were statistical differences for $\mathrm{Ck}-\mathrm{Mb}$ values between the baseline and $10,30,60$, and 300 min points in the MKI group. Mean $\mathrm{Cr}$ values changed in all groups but they were in reference ranges. Glucose increased significantly in study groups between the baseline and 10,30,60, 180, and 300 min points, with the highest values recorded $60 \mathrm{~min}$ after anaesthesia. Similarly, the increase was also significant between the control and study groups. A significant difference was determined in $\mathrm{LDH}$ values between the baseline and 60, 180 and 300 min points in the MK group, with the highest value being at $180 \mathrm{~min}$. The LDH value also increased in the MKI group at 30, 60,180 , and $300 \mathrm{~min}$. There were significant differences in TP measurement in study groups between the baseline and the other anaesthesia intervals. TP values decreased after $10 \mathrm{~min}$ and continued to do so until $300 \mathrm{~min}$ of anaesthesia. TP values were also different between the study and control groups but were in reference range. No significant differences were obtained for cTnI values between the baseline and anaesthesia intervals or between study and control groups.

Histopathological findings. Normal and apoptopic total cell numbers were examined in the liver, brain, and heart and comparisons were made between the groups (Figs 1, 2, and 3). Normal and apoptopic cell numbers determined by the TUNEL method are given in Table 4. 


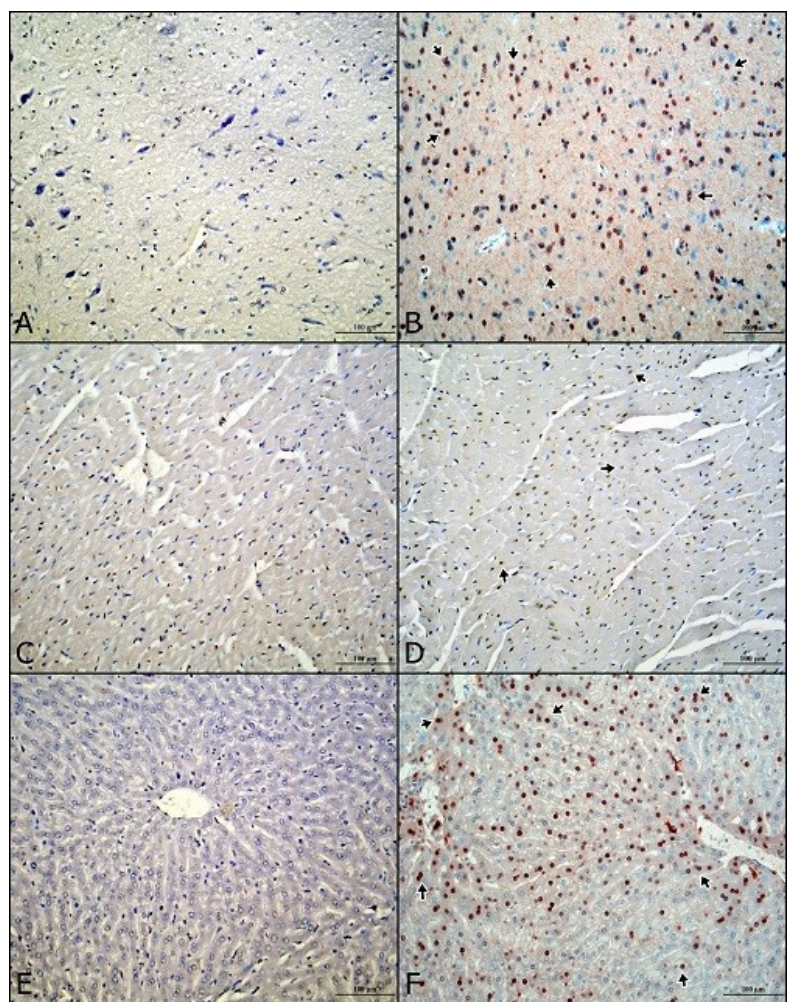

Fig. 1. Immunohistochemical findings of $M$ and control groups A - control, brain, B - M group, glia and neuron cells (arrows), $\mathrm{C}$ - control, myocardium $\mathrm{D}-\mathrm{M}$ group, myocardium and myocardial cells (arrows), E - control, liver, F - M group, liver, hepatocytes (arrows), Bar $-100 \mu \mathrm{m}$

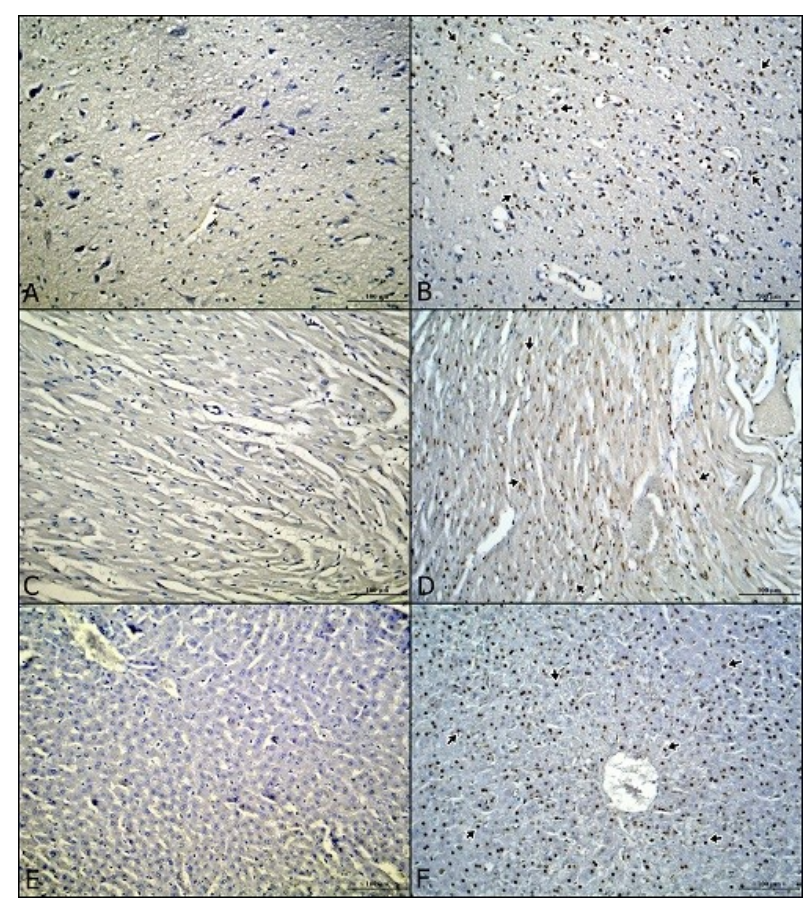

Fig. 2. Immunohistochemical findings of $M K$ and control groups A - control, brain, B - MK group, glia and neuron cells (arrows), C control, myocardium, D - MK group, myocardium and myocardial cells (arrows), E - control, liver, F - MK group, liver, hepatocytes (arrows), Bar $-100 \mu \mathrm{m}$

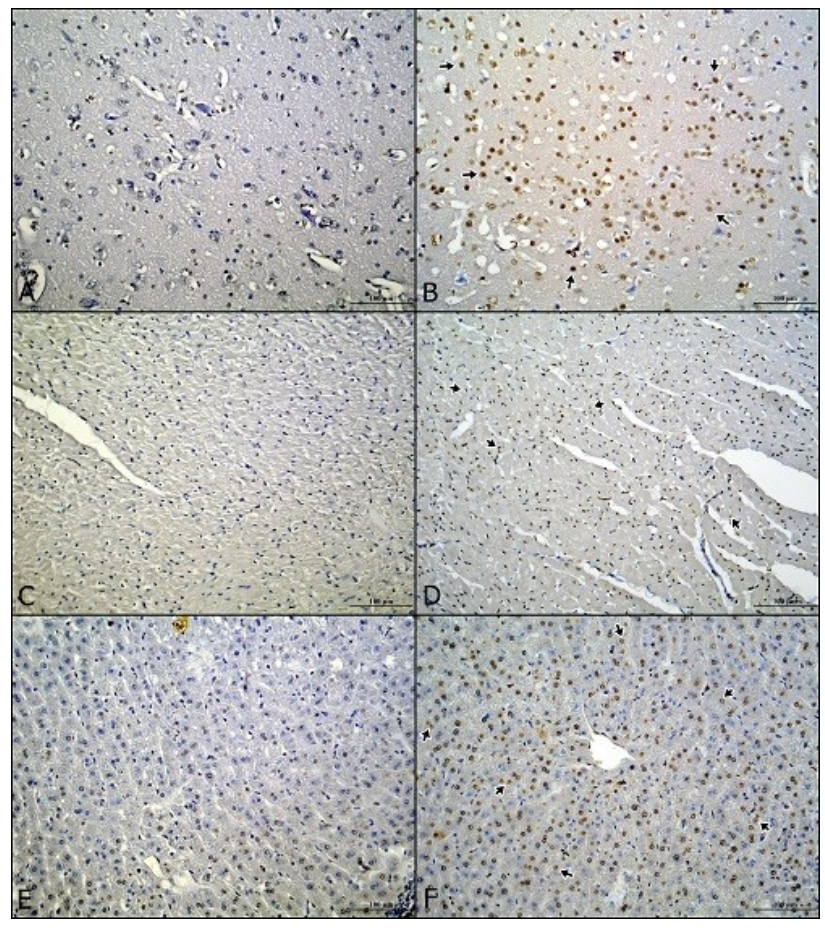

Fig. 3. Immunohistochemical findings of $\mathrm{MKI}$ and control groups A - control, brain, B - MKI group, glia and neuron cells (arrows), C - control, myocardium, D - MKI group, myocardium and myocardial cells (arrows), E - control, liver, F - MKI group, liver, hepatocytes (arrows), Bar $-100 \mu \mathrm{m}$

Histopathological examinations of the liver revealed significant differences between the $\mathrm{M}$ and $\mathrm{MK}$ groups in apoptopic and total cell numbers. Apoptopic cell numbers were higher in the MK group than in the $\mathrm{M}$ group. Apoptotic brain tissue cell numbers were significantly different between the MK, MKI and control groups. Also apoptotic cell numbers were higher in study groups than control groups.

There were statistical differences in apoptopic heart muscle cell numbers between the $\mathrm{M}$ and MKI groups and between the MK and MKI groups. Apoptopic cell numbers were the lowest in the MKI group. Significant difference was also present between the $\mathrm{M}, \mathrm{MK}$ and control groups. Evident vascularisation with hyperaemia was determined by macroscopic and microscopic examinations in the $M$ group in the heart muscle, liver, and particularly in the central nervous system. No abnormal findings were encountered for the other organs examined which were the stomach, intestine, spleen, lungs, adrenal gland, kidneys, pancreas, and lymph nodes.

Histological examination of the organs of study group rabbits revealed focal haemorrhages in a few areas and hyperaemia of the vessels of the liver, heart muscle, and particularly the central nervous system. No significant abnormal lesions were found in the tissues of control group rabbits. 
Table 1. Descriptive statistics for clinical findings $(M \pm S D)$ over the time intervals of the groups

\begin{tabular}{|c|c|c|c|c|c|c|c|}
\hline Values & Group & $0 \mathrm{~min}$ & $10 \mathrm{~min}$ & $30 \mathrm{~min}$ & $60 \mathrm{~min}$ & $180 \mathrm{~min}$ & $300 \mathrm{~min}$ \\
\hline \multirow[t]{4}{*}{ BT $\left({ }^{\circ} \mathrm{C}\right)$} & $\mathrm{M}$ & $39.03 \pm 0.48$ & $38.41 \pm 0.92^{*}$ & $38.03 \pm 1.04^{*}$ & $38.10 \pm 0.99^{*}$ & $37.82 \pm 1.60^{*}$ & $38.25 \pm 0.77^{*}$ \\
\hline & MK & $38.37 \pm 0.75$ & $37.85 \pm 0.70^{* 1}$ & $37.25 \pm 0.96^{* * * 1}$ & $37.12 \pm 0.92^{*, * 1}$ & $37.00 \pm 0.95^{*, * 1}$ & $37.22 \pm 0.74^{*, * 1}$ \\
\hline & MKI & $38.83 \pm 0.90$ & $36.63 \pm 0.98^{*, * 1}$ & $36.13 \pm 1.13^{*, * 1}$ & $35.52 \pm 1.60^{*, * 1}$ & $36.27 \pm 1.70^{* * * 1}$ & $36.88 \pm 1.57^{*, * 1}$ \\
\hline & Control & $38.70 \pm 0.57$ & $38.80 \pm 0.15$ & $38.37 \pm 0.20$ & $38.22 \pm 0.27$ & $38.25 \pm 0.23$ & $37.90 \pm 0.25$ \\
\hline \multirow[t]{4}{*}{$\mathrm{RR}(\min )$} & M & $122.25 \pm 34.39$ & $79.75 \pm 23.38^{*, *{ }^{*}}$ & $71.62 \pm 15.90^{*, * 1}$ & $76.75 \pm 25.38^{*, * 1}$ & $86.00 \pm 29.33^{*}$ & $97.75 \pm 39.62^{*}$ \\
\hline & MK & $142.75 \pm 27.81$ & $87.50 \pm 42.15^{*, *}$ & $57.00 \pm 24.49^{*, * 1}$ & $57.00 \pm 13.52^{*, *}$ & $64.87 \pm 23.64^{*, * 1}$ & $87.50 \pm 44.88^{*}$ \\
\hline & MKI & $117.75 \pm 13.66$ & $39.62 \pm 16.51^{*, *}$ & $43.75 \pm 14.75^{*, * 1}$ & $57.50 \pm 22.72^{*, * 1}$ & $80.75 \pm 21.08^{*}$ & $94.5 \pm 23.99^{*}$ \\
\hline & Control & $136.00 \pm 18.14$ & $123.50 \pm 18.03$ & $101.50 \pm 20.47$ & $95.00 \pm 17.13$ & $100.00 \pm 16.90$ & $108.5 \pm 24.30$ \\
\hline \multirow{4}{*}{$\begin{array}{l}\text { Heart rate } \\
\text { (beat } / \mathrm{min} \text { ) }\end{array}$} & M & $259.30 \pm 38.9$ & $236.40 \pm 35.40$ & $273.10 \pm 23.70$ & $255.50 \pm 30.40$ & $272.50 \pm 24.30$ & $268.5 \pm 35.80$ \\
\hline & MK & $258.60 \pm 55.4$ & $260.60 \pm 23.60$ & $252.40 \pm 39.70$ & $206.50 \pm 23.30^{*}$ & $267.50 \pm 35.40$ & $251.5 \pm 31.60$ \\
\hline & MKI & $276.90 \pm 49.5$ & $253.60 \pm 47.20$ & $232.80 \pm 42.30^{*}$ & $264.00 \pm 38.60$ & $284.50 \pm 21.90$ & $298.0 \pm 37.10$ \\
\hline & Control & $235.60 \pm 44.4$ & $301.80 \pm 11.90$ & $290.40 \pm 31.80$ & $294.00 \pm 42.00$ & $285.80 \pm 20.00$ & $283.5 \pm 29.80$ \\
\hline
\end{tabular}

${ }^{*}$ Comparison between anaesthetic intervals at the same line $(\mathrm{P}<0.05) .{ }^{* 1}-$ statistical significance between study group and control group in the same column $(\mathrm{P}<0.05)$. $\mathrm{BT}$ - body temperature, $\mathrm{RR}$ - respiration rate, $\mathrm{HR}$ - heart rate, $\mathrm{M}$ - midazolam, MK - midazolam-ketamine, MKI - midazolam-ketamine-isoflurane

Table 2. Blood gas values of each group and their statistical significance $(\mathrm{M} \pm \mathrm{SD})$

\begin{tabular}{|c|c|c|c|c|c|c|c|}
\hline Values & Group & $0 \min$ & $10 \mathrm{~min}$ & $30 \mathrm{~min}$ & $60 \mathrm{~min}$ & $180 \mathrm{~min}$ & $300 \mathrm{~min}$ \\
\hline \multirow[t]{4}{*}{$\mathrm{pH}$} & $\mathrm{M}$ & $7.39 \pm 0.06$ & $7.38 \pm 0.06$ & $7.39 \pm 0.10^{* 1}$ & $7.33 \pm 0.08^{* 1}$ & $7.39 \pm 0.07$ & $7.40 \pm 0.08$ \\
\hline & MK & $7.41 \pm 0.08$ & $7.39 \pm 0.08$ & $7.40 \pm 0.06$ & $7.35 \pm 0.04^{* 1}$ & $7.35 \pm 0.07$ & $7.36 \pm 0.07$ \\
\hline & MKI & $7.43 \pm 0.08$ & $7.24 \pm 0.13^{*, * 1}$ & $7.32 \pm 0.11^{*, * 1}$ & $7.24 \pm 0.11^{*, * 1}$ & $7.32 \pm 0.08^{*, * 1}$ & $7.34 \pm 0.08^{*}$ \\
\hline & Control & $7.41 \pm 0.09$ & $7.38 \pm 0.07$ & $7.48 \pm 0.06$ & $7.49 \pm 0.06$ & $7.45 \pm 0.07$ & $7.43 \pm 0.06$ \\
\hline \multirow{4}{*}{$\begin{array}{l}\mathrm{PCO}_{2} \\
(\mathrm{~mm} / \mathrm{Hg})\end{array}$} & M & $32.25 \pm 8.72$ & $33.13 \pm 8.67^{* * * 1}$ & $34.70 \pm 7.90^{*, * 1}$ & $35.1 \pm 7.02^{*, * 1}$ & $34.39 \pm 8.30^{*}$ & $35.56 \pm 6.51^{*, * 1}$ \\
\hline & MK & $29.69 \pm 8.38$ & $30.00 \pm 5.64^{*}$ & $39.29 \pm 5.44^{*, * 1}$ & $41.10 \pm 5.50^{*, * 1}$ & $35.74 \pm 8.52^{*}$ & $34.55 \pm 8.64^{*}$ \\
\hline & MKI & $17.96 \pm 2.05$ & $38.28 \pm 10.52^{*, * 1}$ & $34.48 \pm 10.07^{*, * 1}$ & $29.11 \pm 6.32^{*, * 1}$ & $24.15 \pm 6.62^{*}$ & $21.31 \pm 8.12^{* 1}$ \\
\hline & Control & $21.81 \pm 5.07$ & $21.58 \pm 4.83$ & $19.48 \pm 2.86$ & $18.65 \pm 3.01$ & $17.06 \pm 3.85$ & $14.68 \pm 2.65$ \\
\hline \multirow{4}{*}{$\begin{array}{l}\mathrm{PO}_{2} \\
(\mathrm{~mm} / \mathrm{Hg})\end{array}$} & $\mathrm{M}$ & $76.74 \pm 17.88^{* 1}$ & $74.01 \pm 11.93^{* 1}$ & $72.94 \pm 12.83^{*_{1}}$ & $69.58 \pm 13.25^{* 1}$ & $61.31 \pm 14.47^{*, * 1}$ & $68.04 \pm 21.59^{* 1}$ \\
\hline & MK & $68.41 \pm 21.53^{* 1}$ & $65.91 \pm 18.01^{* 1}$ & $54.99 \pm 9.63^{*, * 1}$ & $64.18 \pm 11.82^{* 1}$ & $50.48 \pm 10.06^{*, * 1}$ & $48.64 \pm 11.96^{*, * 1}$ \\
\hline & MKI & $73.25 \pm 10.69^{* 1}$ & $88.75 \pm 13.23^{* 1}$ & $63.75 \pm 24.30^{* 1}$ & $46.13 \pm 13.49^{*,{ }^{*} 1}$ & $46.88 \pm 10.86^{*, * 1}$ & $41.88 \pm 13.14^{*,{ }^{*} 1}$ \\
\hline & Control & $48.63 \pm 6.25$ & $46.88 \pm 5.05$ & $46.13 \pm 7.06$ & $44.25 \pm 6.73$ & $48.50 \pm 9.32$ & $49.50 \pm 8.97$ \\
\hline \multirow{4}{*}{$\begin{array}{l}\mathrm{HCO}_{3} \\
\mathrm{Meq} / \mathrm{L}\end{array}$} & M & $18.93 \pm 3.75$ & $19.70 \pm 3.61$ & $20.54 \pm 3.06$ & $20.49 \pm 3.39$ & $20.38 \pm 4.92$ & $21.54 \pm 3.78^{* 1}$ \\
\hline & MK & $20.61 \pm 5.44$ & $20.51 \pm 4.22$ & $23.86 \pm 2.39$ & $23.39 \pm 1.83^{* 1}$ & $21.83 \pm 2.78^{* 1}$ & $20.29 \pm 2.00^{* 1}$ \\
\hline & MKI & $17.08 \pm 3.29$ & $23.34 \pm 6.06^{* 1}$ & $17.68 \pm 5.72$ & $14.09 \pm 3.83^{*}$ & $13.63 \pm 1.71^{*}$ & $13.19 \pm 2.35^{*}$ \\
\hline & Control & $17.53 \pm 2.83$ & $16.35 \pm 2.12$ & $19.05 \pm 2.65$ & $18.99 \pm 2.67$ & $15.43 \pm 2.98$ & $13.99 \pm 1.47$ \\
\hline \multirow{4}{*}{$\begin{array}{l}\mathrm{tCO}_{2} \\
\mathrm{mM} \mathrm{L} /\end{array}$} & M & $19.8 \pm 3.99^{* 1}$ & $20.73 \pm 3.68^{* 1}$ & $21.61 \pm 3.21$ & $20.63 \pm 5.12^{* 1}$ & $21.66 \pm 4.34^{* 1}$ & $22.55 \pm 3.93^{* 1}$ \\
\hline & MK & $19.3 \pm 6.64^{* 1}$ & $20.4 \pm 5.55^{* 1}$ & $26.15 \pm 3.09^{*, * 1}$ & $25.84 \pm 2.19^{*, * 1}$ & $21.96 \pm 4.55^{* 1}$ & $20.84 \pm 2.73^{* 1}$ \\
\hline & MKI & $12.38 \pm 3.11$ & $29.0 \pm 9.00^{*}$ & $17.75 \pm 8.27^{*}$ & $13.86 \pm 4.96$ & $11.0 \pm 3.93$ & $9.0 \pm 3.12$ \\
\hline & Control & $14.0 \pm 3.46$ & $12.99 \pm 2.72$ & $15.05 \pm 2.96$ & $14.76 \pm 2.86$ & $11.14 \pm 4.25$ & $8.19 \pm 2.59$ \\
\hline \multirow{4}{*}{$\begin{array}{l}\mathrm{BE} \\
\mathrm{mM} / \mathrm{L}\end{array}$} & M & $-4.13 \pm 3.34$ & $-3.49 \pm 3.78^{* 1}$ & $-3.01 \pm 3.66$ & $-4.50 \pm 5.39$ & $-2.74 \pm 4.55^{* 1}$ & $-2.13 \pm 4.44^{* 1}$ \\
\hline & MK & $-3.2 \pm 7.40$ & $-4.35 \pm 4.99^{* 1}$ & $0.03 \pm 3.38^{* 1}$ & $-1.94 \pm 1.73^{* 1}$ & $-4.41 \pm 3.44^{* 1}$ & $-5.43 \pm 2.79^{* 1}$ \\
\hline & MKI & $-9.35 \pm 4.29$ & $-1.65 \pm 6.73^{*, * 1}$ & $-8.55 \pm 7.49^{* 1}$ & $-13.01 \pm 5.03^{* 1}$ & $-12.93 \pm 2.69$ & $-14.16 \pm 3.30^{*}$ \\
\hline & Control & $-8.45 \pm 3.93$ & $-10.08 \pm 3.02$ & $-6.39 \pm 3.61$ & $-6.46 \pm 3.61$ & $-9.86 \pm 4.49$ & $-12.91 \pm 2.38$ \\
\hline \multirow{4}{*}{$\begin{array}{l}\text { BEecf } \\
\mathrm{mM} / \mathrm{L}\end{array}$} & M & $-5.48 \pm 3.85$ & $-4.66 \pm 4.05^{* 1}$ & $-3.93 \pm 3.71$ & $-5.91 \pm 5.68$ & $-3.86 \pm 4.12^{* 1}$ & $-2.89 \pm 4.63^{* 1}$ \\
\hline & MK & $-5.31 \pm 8.03$ & $-3.70 \pm 6.19^{* 1}$ & $-0.11 \pm 3.70$ & $-1.71 \pm 2.12^{* 1}$ & $-4.16 \pm 3.95^{* 1}$ & $-6.26 \pm 3.37^{* 1}$ \\
\hline & MKI & $-12.09 \pm 4.50$ & $-0.56 \pm 8.10^{*, * 1}$ & $-9.49 \pm 8.61$ & $-14.31 \pm 5.54$ & $-15.10 \pm 3.35$ & $-16.70 \pm 3.37$ \\
\hline & Control & $-10.76 \pm 4.11$ & $-11.71 \pm 3.75$ & $-8.79 \pm 3.79$ & $-8.86 \pm 3.80$ & $-12.96 \pm 4.71$ & $16.18 \pm 2.47$ \\
\hline \multirow{4}{*}{$\begin{array}{l}\mathrm{O}_{2} \mathrm{SAT} \\
\%\end{array}$} & M & $90.98 \pm 9.94$ & $92.65 \pm 3.13$ & $93.56 \pm 5.25^{* 1}$ & $89.95 \pm 6.90^{* 1}$ & $87.83 \pm 8.98$ & $88.41 \pm 14.24$ \\
\hline & MK & $88.09 \pm 15.82$ & $89.09 \pm 9.85$ & $85.04 \pm 10.48$ & $90.33 \pm 2.85$ & $85.18 \pm 6.89$ & $78.45 \pm 14.90$ \\
\hline & MKI & $93.38 \pm 3.66$ & $97.75 \pm 3.49^{* 1}$ & $78.38 \pm 29.51^{*}$ & $74.75 \pm 20.28^{*}$ & $77.63 \pm 15.95^{*}$ & $74.00 \pm 18.44^{*}$ \\
\hline & Control & $82.38 \pm 7.34$ & $80.63 \pm 6.69$ & $82.63 \pm 6.11$ & $81.25 \pm 5.92$ & $83.38 \pm 5.92$ & $84.25 \pm 6.67$ \\
\hline
\end{tabular}

${ }^{*}$ Comparison between anaesthetic intervals at the same line $(\mathrm{P}<0.05) .{ }^{* 1}-$ statistical significance between study group and control group in the same column $(\mathrm{P}<0.05)$. $\mathrm{pH}$ - venous blood $\mathrm{pH}, \mathrm{PCO}_{2}$ - partial venous blood carbon dioxide, $\mathrm{HCO}_{3}$ - bicarbonate, $\mathrm{tCO}_{2}$ - total carbon dioxide, $\mathrm{BE}$ - base excess, $\mathrm{BEecf}$ - extra cellular fluid, $\mathrm{O}_{2} \mathrm{SAT}$ - oxygen saturation, $\mathrm{M}$ - midazolam, MK - midazolam-ketamine, MKI - midazolam-ketamine-isoflurane 
Table 3. Serum biochemical values of each group and their statistical significance $(M \pm S D)$

\begin{tabular}{|c|c|c|c|c|c|c|c|}
\hline Values & Group & $0 \mathrm{~min}$ & $10 \mathrm{~min}$ & $30 \mathrm{~min}$ & $60 \mathrm{~min}$ & $180 \mathrm{~min}$ & $300 \mathrm{~min}$ \\
\hline \multirow{4}{*}{$\begin{array}{l}\text { ALP } \\
(\mathrm{I} / \mathrm{U})\end{array}$} & $\mathrm{M}$ & $5.57 \pm 0.62$ & $5.16 \pm 0.62^{*, * 1}$ & $5.14 \pm 0.62^{*}$ & $5.12 \pm 0.52^{*}$ & $4.84 \pm 0.62^{*}$ & $4.65 \pm 0.78^{*, * 1}$ \\
\hline & MK & $5.92 \pm 0.21$ & $5.54 \pm 0.50^{*}$ & $5.25 \pm 1.04^{*, *{ }^{*} 1}$ & $5.39 \pm 0.80^{*}$ & $5.33 \pm 0.67^{*}$ & $5.59 \pm 0.42^{*}$ \\
\hline & MKI & $5.54 \pm 0.23$ & $4.75 \pm 0.62^{*, * 1}$ & $4.45 \pm 0.70^{*, * 1}$ & $4.5 \pm 0.59^{*, * 1}$ & $4.8 \pm 0.62^{*}$ & $4.67 \pm 1.19^{*}$ \\
\hline & Control & $5.98 \pm 0.03$ & $5.96 \pm 0.09$ & $5.90 \pm 0.10$ & $5.66 \pm 0.20$ & $5.03 \pm 0.36$ & $5.15 \pm 0.38$ \\
\hline \multirow{4}{*}{$\begin{array}{l}\text { ALT } \\
(\mathrm{I} / \mathrm{U})\end{array}$} & M & $41.5 \pm 51.92^{* 1}$ & $44.25 \pm 33.45^{* 1}$ & $35.75 \pm 33.91^{* 1}$ & $36.37 \pm 30.99$ & $31.12 \pm 23.33^{*}$ & $28.25 \pm 22.92^{*}$ \\
\hline & MK & $21.62 \pm 7.50$ & $19.5 \pm 7.17$ & $21.13 \pm 1.24$ & $24.63 \pm 12.14$ & $22.25 \pm 9.57$ & $23.75 \pm 10.60$ \\
\hline & MKI & $22.75 \pm 5.12$ & $18.0 \pm 4.27$ & $19.25 \pm 7.77$ & $21.37 \pm 6.50$ & $24.63 \pm 9.92$ & $34.13 \pm 16.98^{*, * 1}$ \\
\hline & Control & $15.63 \pm 8.61$ & $15.38 \pm 7.48$ & $13.88 \pm 5.48$ & $16.38 \pm 6.23$ & $15.13 \pm 5.05$ & $17.5 \pm 5.48$ \\
\hline \multirow{4}{*}{$\begin{array}{l}\text { AST } \\
(\mathrm{I} / \mathrm{U})\end{array}$} & M & $32.13 \pm 24.52$ & $47.38 \pm 27.07$ & $58.38 \pm 30.61^{*, * 1}$ & $63.13 \pm 30.42^{*,{ }^{*} 1}$ & $77.0 \pm 31.74^{*, * 1}$ & $96.75 \pm 49.55^{*, * 1}$ \\
\hline & MK & $24.13 \pm 9.06$ & $32.63 \pm 9.02$ & $37.38 \pm 14.7$ & $46.63 \pm 18.42^{*}$ & $55.13 \pm 21.67^{*,{ }^{*} 1}$ & $51.63 \pm 16.69^{*}$ \\
\hline & MKI & $22.38 \pm 3.81$ & $39.0 \pm 16.37$ & $38.5 \pm 14.45$ & $62.63 \pm 22.3^{*, * 1}$ & $84.5 \pm 38.92^{*, * 1}$ & $100.38 \pm 67.51^{*, * 1}$ \\
\hline & Control & $14.88 \pm 4.01$ & $17.0 \pm 4.53$ & $18.75 \pm 4.59$ & $23.5 \pm 7.17$ & $22.88 \pm 6.05$ & $35.88 \pm 9.77$ \\
\hline \multirow{4}{*}{$\begin{array}{l}\text { BUN } \\
(\mathrm{mg} / \mathrm{d} l)\end{array}$} & M & $18.19 \pm 4.13$ & $21.30 \pm 4.13^{*}$ & $19.39 \pm 4.09$ & $20.81 \pm 4.56$ & $21.31 \pm 4.92^{*}$ & $19.86 \pm 3.35$ \\
\hline & MK & $18.14 \pm 3.61$ & $18.88 \pm 4.02$ & $18.96 \pm 5.21$ & $20.09 \pm 5.66$ & $22.61 \pm 3.03^{*}$ & $24.55 \pm 3.69^{*, * 1}$ \\
\hline & MKI & $16.04 \pm 5.76$ & $13.44 \pm 2.79^{*, * 1}$ & $13.18 \pm 2.89^{*}$ & $13.29 \pm 2.89^{*, * 1}$ & $14.21 \pm 3.50^{* 1}$ & $15.94 \pm 4.60^{* 1}$ \\
\hline & Control & $19.24 \pm 4.60$ & $18.58 \pm 4.70$ & $20.54 \pm 4.73$ & $20.35 \pm 3.88$ & $19.11 \pm 1.07$ & $19.29 \pm 1.64$ \\
\hline \multirow{4}{*}{$\begin{array}{l}\text { Ck-Mb } \\
(\mathrm{I} / \mathrm{U})\end{array}$} & M & $480.15 \pm 196.42$ & $353.72 \pm 107.62$ & $424.5 \pm 69.14$ & $436.5 \pm 69.57$ & $387.02 \pm 156.50$ & $319.41 \pm 141.05$ \\
\hline & MK & $645.80 \pm 640.59$ & $655.87 \pm 695.48$ & $646.91 \pm 743.31$ & $626.88 \pm 687.33$ & $660.12 \pm 511.89$ & $458.96 \pm 202.05$ \\
\hline & MKI & $804.98 \pm 664.33^{* 1}$ & $325.53 \pm 136.25^{*}$ & $348.73 \pm 133.76^{*}$ & $612.86 \pm 203.60^{*}$ & $645.60 \pm 207.90^{* 1}$ & $421.97 \pm 150.94^{*}$ \\
\hline & Control & $376.06 \pm 99.0$ & $387.16 \pm 106.11$ & $321.61 \pm 77.29$ & $446.67 \pm 46.5$ & $527.4 \pm 66.91$ & $479.96 \pm 27.63$ \\
\hline \multirow{4}{*}{$\begin{array}{l}\mathrm{Cr} \\
(\mathrm{mg} / \mathrm{dL})\end{array}$} & M & $1.05 \pm 0.21$ & $1.02 \pm 0.24$ & $1.12 \pm 0.13$ & $1.02 \pm 0.22$ & $1.0 \pm 0.34$ & $1.0 \pm 0.25$ \\
\hline & MK & $1.08 \pm 0.18$ & $1.01 \pm 0.16$ & $0.93 \pm 0.26^{*}$ & $0.93 \pm 0.14^{*}$ & $0.98 \pm 0.06$ & $0.98 \pm 0.17$ \\
\hline & MKI & $1.3 \pm 0.40$ & $1.14 \pm 0.16^{*}$ & $1.09 \pm 0.16^{*}$ & $1.05 \pm 0.20^{*}$ & $1.05 \pm 0.18^{*, * 1}$ & $1.09 \pm 0.34^{*}$ \\
\hline & Control & $1.02 \pm 0.13$ & $1.06 \pm 0.16$ & $1.16 \pm 0.16$ & $0.93 \pm 0.14$ & $0.79 \pm 0.08$ & $0.88 \pm 0.08$ \\
\hline \multirow{4}{*}{$\begin{array}{l}\text { Glu } \\
(\mathrm{mg} / \mathrm{dL})\end{array}$} & M & $141.88 \pm 23.86$ & $244.63 \pm 109.60^{*, * 1}$ & $264.88 \pm 66.63^{*, * 1}$ & $292.63 \pm 62.58^{*, * 1}$ & $233.00 \pm 112.56^{*, * 1}$ & $22.63 \pm 137.03^{*, * 1}$ \\
\hline & MK & $128.13 \pm 45.36$ & $183.25 \pm 46.75^{*}$ & $238.75 \pm 72.81^{*, * 1}$ & $267.00 \pm 77.05^{*, * 1}$ & $220.38 \pm 115.30^{*, * 1}$ & $208.13 \pm 149.34^{*, * 1}$ \\
\hline & MKI & $81.75 \pm 32.81^{*}$ & $187.38 \pm 4.70^{1 *}$ & $170.75 \pm 39.47^{*}$ & $232.00 \pm 51.61^{*, * 1}$ & $219.13 \pm 53.73^{*, * 1}$ & $138.88 \pm 43.87^{*}$ \\
\hline & Control & $125.25 \pm 34.62$ & $156.88 \pm 68.92$ & $168.88 \pm 63.33$ & $138.88 \pm 13.10$ & $132.00 \pm 14.59$ & $125.63 \pm 16.06$ \\
\hline \multirow{4}{*}{$\begin{array}{l}\text { LDH } \\
(\mathrm{I} / \mathrm{U})\end{array}$} & M & $393.13 \pm 167.68$ & $355.38 \pm 121.88$ & $535.38 \pm 252.56$ & $474.25 \pm 169.53$ & $378.75 \pm 133.05$ & $321.13 \pm 129.96$ \\
\hline & MK & $395.00 \pm 217.94$ & $395.25 \pm 158.46$ & $473.00 \pm 225.60^{* 1}$ & $592.88 \pm 204.35^{*, * 1}$ & $865.25 \pm 352.50^{*, * 1}$ & $644.25 \pm 247.82^{*}$ \\
\hline & MKI & $404.38 \pm 120.31$ & $365.25 \pm 151.04$ & $469.50 \pm 175.09^{*, * 1}$ & $866.25 \pm 346.09^{*,{ }^{*} 1}$ & $1263.13 \pm 895.82^{*, * 1}$ & $1389.50 \pm 106.49^{*, *{ }_{1}}$ \\
\hline & Control & $105.88 \pm 52.74$ & $129.13 \pm 64.27$ & $136.63 \pm 76.81$ & $280.00 \pm 98.97$ & $297.88 \pm 74.27$ & $367.38 \pm 109.85$ \\
\hline \multirow{4}{*}{$\begin{array}{l}\mathrm{TP} \\
(\mathrm{g} / \mathrm{dL})\end{array}$} & M & $7.25 \pm 1.28$ & $6.60 \pm 1.52^{*}$ & $6.65 \pm 1.42^{*}$ & $6.61 \pm 1.05^{*}$ & $6.16 \pm 1.59^{*}$ & $6.10 \pm 1.61^{*}$ \\
\hline & MK & $6.85 \pm 0.62$ & $6.41 \pm 0.64^{*, * 1}$ & $5.94 \pm 1.17^{*, * 1}$ & $6.0 \pm 1.16^{*}$ & $6.28 \pm 0.70$ & $6.13 \pm 0.76^{*}$ \\
\hline & MKI & $7.0 \pm 0.76$ & $5.75 \pm 0.48^{*}$ & $5.46 \pm 0.79^{*}$ & $5.56 \pm 0.52^{*}$ & $5.66 \pm 0.68^{*}$ & $5.41 \pm 1.25^{*}$ \\
\hline & Control & $7.45 \pm 0.68$ & $7.26 \pm 0.54$ & $7.11 \pm 0.67$ & $6.31 \pm 0.47$ & $5.99 \pm 0.35$ & $5.71 \pm 0.32$ \\
\hline \multirow{4}{*}{$\begin{array}{l}\mathrm{cTnl} \\
(\mu \mathrm{g} / \mathrm{dL})\end{array}$} & M & $0.1 \pm 0.07$ & $0.07 \pm 0.00$ & $0.10 \pm 0.05$ & $0.16 \pm 0.07$ & $0.23 \pm 0.12$ & $0.27 \pm 0.09$ \\
\hline & MK & $0.07 \pm 0.00$ & $0.07 \pm 0.01$ & $0.07 \pm 0.00$ & $0.08 \pm 0.02$ & $0.11 \pm 0.12$ & $0.13 \pm 0.08$ \\
\hline & MKI & $0.09 \pm 0.04$ & $0.21 \pm 0.31$ & $0.39 \pm 0.84$ & $0.47 \pm 0.96$ & $0.76 \pm 1.32^{* 1}$ & $1.26 \pm 2.29^{*, * 1}$ \\
\hline & Control & $0.07 \pm 0.00$ & $0.07 \pm 0.01$ & $0.1 \pm 0.05$ & $0.12 \pm 0.10$ & $0.11 \pm 0.06$ & $0.11 \pm 0.07$ \\
\hline
\end{tabular}

${ }^{*}$ Comparison between anaesthetic intervals at the same line $(\mathrm{P}<0.05) .{ }^{* 1}-$ statistical significance between study group and control group in the same column $(\mathrm{P}<0.05)$. ALP - alkaline phosphatase, ALT - alanine aminotransferase, AST - aspartate aminotransferase, BUN - blood urea nitrogen, $\mathrm{Ck}-\mathrm{Mb}$ - creatine kinase-Mb, Cr - creatine, Glu - glucose, LDH - lactate dehydrogenase, TP - total protein, cTnl - cardiac troponin, M - midazolam, MK - midazolam-ketamine, MKI - midazolam-ketamineisoflurane 
Table 4. Histopathological evaluation of apoptotic and normal cell numbers of each group (median (min-max)

\begin{tabular}{llll}
\hline Tissue & Group & Apoptopic cell & Normal cell \\
\hline \multirow{4}{*}{ Liver } & M & $306.0(90-430)^{*}$ & $341.0(257-545)$ \\
& MK & $470.0(422-572)^{*}$ & $323.0(297-342)$ \\
& MKI & $398.0(141-520)$ & $278.0(156-463)$ \\
& Control & $313.0(176-512)$ & $405.0(327-510)$ \\
& & & \\
\multirow{5}{*}{ Brain } & M & $232.5(151-343)$ & $249.0(179-282)$ \\
& MK & $246.5(221-441)^{* *}$ & $200.5(178-283)$ \\
& MKI & $259.0(120-412)^{* *}$ & $130.5(120-198)$ \\
& Control & $151.0(120-210)$ & $247.0(163-305)$ \\
& & & \\
Myocardium & MK & $354.0(266-372)^{* * * *}$ & $213.0(197-220)$ \\
& MKI & $197.0(135-248)$ & $302.5(203-329)$ \\
& Control & $184.5(152-224)$ & $279.0(196-354)$ \\
\hline
\end{tabular}

** Difference between the groups in the same column is statistically significant $(\mathrm{P}<0.001), *$ Difference between the experiment and control group is statistically significant $(\mathrm{P}<0.05)$. M - midazolam, MK - midazolam-ketamine, MKI - midazolam-ketamine-isoflurane

\section{Discussion}

It was determined that midazolam, ketamine, and isoflurane anaesthesia caused changes in rabbit organs. In the clinical analysis, after anaesthesia, the low BT and RR were found to be significant statistically $(\mathrm{P}<0.05)$ (Table 1). The drop in body temperature observed in the experimental groups $10 \mathrm{~min}$ after anaesthetic application is the result of suppression of the thermoregulation centre by the anaesthetics. Environmental temperature and progressing vasodilatation contributed to the development of hypothermia $(15,16,18)$.

When venous blood gases were evaluated, the determined changes were within references ranges (2). Especially in the $\mathrm{M}$ and $\mathrm{MK}$ groups at 30 and $60 \mathrm{~min}$, and in the MKI group except for at $300 \mathrm{~min}, \mathrm{pH}$ values decreased significantly at the other time points, which was compatible with the increase in $\mathrm{PCO}_{2}$ at the same times and this condition supported a suspicion of moderate respiratory acidosis. In the study groups, changes were particularly pronounced at 30 and $60 \mathrm{~min}$. Respiratory acidosis was present as reported in the literature which seems to occur as a result of suppression of the respiratory system by anaesthetics $(24,25)$.

The $\mathrm{Ck}-\mathrm{Mb}$ and $\mathrm{cTnI}$ values are indicators for degeneration of the heart muscle as a result of arrhythmia occurring during anaesthesia (27). In the present study, $\mathrm{Ck}-\mathrm{Mb}$ and $\mathrm{cTnI}$ values in each study group were different at different sampling times. Differences observed in the $\mathrm{Ck}-\mathrm{Mb}$ and $\mathrm{cTnI}$ values are believed to be the result of a fall in the heart rate caused by the anaesthetic agents and this occurred due to hypoxia, which was determined by blood gas analysis. Blood gas analysis showed that in experimental groups, starting from $10 \mathrm{~min}, \mathrm{PCO}_{2}$ generally increased and
$\mathrm{PO}_{2}$ decreased and these were the evidence of hypoxia (Table 2). But in the MKI group, after $30 \mathrm{~min}, \mathrm{PCO}_{2}$ values did not increase due to $\mathrm{O}_{2}$ reinforcement. Furthermore, Glu and LDH values were also higher than their reference ranges and these increases also supported hypoxia (Table 3).

During anaesthesia, due to anaesthetic agents used, respiratory and cardio vascular system depression occurs and this reduces tissue perfusion. After deterioration of tissue perfusion and slowing circulation, the anaerobic metabolism starts to produce lactate. Increased lactate causes an increase in blood glucose level. In this situation, the liver produces glucose more than normal and LDH values rise (10). Furthermore, the reason for an intensification in $\mathrm{LDH}$ and AST activity is an increase in the concentrations of anaesthetic agents in the liver and subsequently developing uplift in the cellular enzyme secretion.

In general, the presence of high enzyme values compared to control values proved the effects of anaesthetic agents on Glu, LDH, and AST. On the other hand, apart from Glu, LDH, and AST values, biochemical parameters were within reference ranges (24). In the MKI group, LDH values $(1,263.1 \pm$ $895.8 \mathrm{IU} / \mathrm{L}$ and $1,389.5 \pm 1,060.5 \mathrm{IU} / \mathrm{L})$ obtained at 180 and $300 \mathrm{~min}$, respectively were higher than those of the same group at 0 min and other groups at the same time and this shows the important effect of the triple combination (the MKI group) on LDH. Although LDH values in other groups were in the reference ranges, when the values were compared to their baseline values they were higher. It can be speculated that these increases could be due to organ hyperaemia occurred in the study groups. When the triple combination was used, it is believed to have had a more toxic effect at organ level on LDH value than other groups. Several anaesthetic agents are metabolised in the liver and removed from the body through the kidneys. Therefore, anaesthetic agents have more toxic effects on the liver and kidneys (17). Changes caused by midazolam and ketamine as a result of their metabolism in the liver were compatible with the literature data consulted $(13,25)$.

In the present study, apoptotic brain tissue cell numbers were different in MK, MKI, and control groups. The number of apoptotic cells in the MKI group was higher than in the $\mathrm{M}, \mathrm{MK}$, and control groups. However, the differences were only statistically significant between the MK and MKI groups $(\mathrm{P}<0.05)$. In rats, intratracheal usage of $5 \%$ ketamine caused neurological changes (1). But in humans, the usage of ketamine caused none (4). The neurodegenerative changes and apoptosis in nerve tissue determined in the present study were in agreement with the results obtained from rodents. Toxic effects of ketamine on the nervous system in these animals may manifest even at normal parenteral doses. Furthermore, in rabbits receiving midazolam and preservative-free midazolam, evident histopathological 
changes were also observed under light and fluorescence microscopy (12). Midazolam has neurotoxic properties when administered neuroaxially in vivo. It also induces neurodegeneration with other general anaesthetics in neonatal animals. Especially in studies on apoptosis, anaesthetic agents used at different concentrations inhibit mitochondrial activity and induce apoptosis (26).

In the present study, it has also been shown that ketamine, midazolam, and triple combination anaesthesia may cause apoptosis, suggested by the lesser extent of apoptosis in the controls, which supports the above studies. But, in different organs, there are different effects. In the MK group the apoptotic effects on the liver cells were especially more pronounced than those of the midazolam study group. A greater number of apoptotic cells in the liver, especially in the MK group, the potential to be due to metabolism of midazolam and ketamine in the liver, participation of inflammatory mediators in portal circulation together with a greater amount of catecholamine, and attribution to an increase in synergistic effects of these two anaesthetic agents, all these circumstances showed compatibility with the literature data $(5,13)$. In addition, increases in the enzyme values obtained in the present study support histopathological apoptotic changes in the liver.

Oxygen saturation may be reduced if the combination of anaesthetic agents is appropriate to provoke this. Diminished oxygen saturation results in hypoxia and hypothermia leading to neuronal cell death in the brain (20). In addition, anaesthetic agents that have water solubility such as midazolam, and anaesthetics that can pass easily into fatty tissue, other tissues, and cross the blood brain barrier have high affinities to GABA and NMDA receptors in CNS, so may cause increase in apoptosis.

The increase in apoptosis in brain cells in this study was in agreement with this explanation and the literature data $(5,11,23)$. Changes in oxygen saturations and body temperatures, which are affected by the anaesthetic agents used, probably increase the rate of apoptosis and apoptotic cell count. However, the highest number of apoptotic cells being in the MKI group supported the theory that it was the effect of oxygen saturation. Furthermore, ketamine and isoflurane support cerebral blood flow and increased intracranial pressure, supporting increased cerebral metabolism with increased blood flow. Their combination has also been reported to block the calcium channels, reduce the intracellular calcium flux, and prevent the release of neurotransmitter substances on glutamate NMDA receptors, thus, it shows a neuroprotective effect in the brain $(11,28)$. The fact that the apoptotic cell number in the brain differs from that of control groups, especially in the MK and MKI groups, suggests that ketamine and isoflurane may cause apoptosis in brain cells. In histopathological examinations in the course of the present study, hyperaemia and focal bleeding in the cerebral vessels of experimental groups were in agreement with the literature data insofar as they were from increasing intracranial pressure and blood flow (11, 28). Furthermore, the histopathological findings obtained by Auroy et al. (3) investigating the neurotoxic effects of midazolam were compatible with the histopathological findings of the present study.

Isoflurane inhalation anaesthesia inflicted a lower apoptotic cell number in the myocardium than in the $\mathrm{M}$ and MK groups. The rise in apoptotic cell numbers detected in the heart supported the assumption that anaesthetic agents exert such effects on the cardiovascular system.

The increased number of apoptotic cells in the $\mathrm{M}$ and MK groups led to a more pronounced negative chronotropic effect on the cardiovascular system of midazolam and ketamine, whereas in group MKI, the presence of lower numbers of apoptotic cells compared to groups $\mathrm{M}$ and $\mathrm{MK}$ was thought to be due to oxygen support during anaesthesia.

In conclusion, the anaesthetic agents used may normally increase the occurrence of programmed apoptosis in body systems. The MKI anaesthesia combination used in the study group was found to cause less cell destruction in general than in MK group and anaesthetic agents used in the other experimental groups. Therefore, when the advantages such as safe entry to anaesthesia, prolongation of anaesthesia, and oxygen support are taken into account, it was observed that MKI combination anaesthesia caused less toxic effect in rabbits than midazolam and midazolamketamine, and provided safer anaesthesia. Considering this situation, it is suggested that the selection of anaesthesia combinations is important and the studies on this subject are inadequate, and it is needful to continue them.

Conflict of Interests Statement: The authors declare that there is no conflict of interests regarding the publication of this article.

Financial Disclosure Statement: This Project was supported by the Scientific and Technological Research Council of Turkey (TUBITAK), (Project code: 1130733).

Animal Rights Statements: The study was approved by the Erciyes University Local Ethics Committee (ERU-HADYEK, decision no: 13/37).

\section{References}

1. Amiot M.J., Fleuriet A., Macheix J.J.: Importance and evolution of phenolic compounds in olive during growth and maturation. J Agric Food Chem 1986, 34, 823-826.

2. Ardiaca M., Bonvehi C., Montesinos A.: Point-of-care blood gas and electrolyte analysis in rabbits. Vet Clin North Am Exot Anim Pract 2013, 16, 175-195. 
3. Auroy P., Schoeffler P., Maillot C., Haberer J.P., Woda A.: Intrathecal tolerability of midazolam. Histological study. Ann Fr Anesth Reanim 1988, 7, 81-82.

4. Bion J.F.: Infusion analgesia for acute war injuries. A comparison of pentozocaine and ketamine. Anaesthesia 1984, 39, 560-564

5. Braun S., Gaza N., Werdehausen R., Hermanns H., Bauer I., Durieux M.E., Hollmann M.W., Stevens M.F.: Ketamine induces apoptosis via the mitochondrial pathway in human lymphocytes and neuronal cells. Br J Anaesth 2010, 105, 347-354.

6. But K.A., Durmus M., Toprak H.I., Ozturk E., Demirbilek S., Ersoy M.O.: Hemodynamic, hepatorenal, and postoperative effects of desflurane-fentanyl and midazolam-fentanyl anesthesia in coronary artery bypass surgery. J Cardiothorac Vasc Anesth 2005, 19, 597-602.

7. Cattano D., Young C., Straiko M.M., Olney J.W.: Subanesthetic doses of propofol induce neuroapoptosis in the infant mouse brain. Anesth Analg 2008, 106, 1712-1714.

8. Close B., Banister K., Baumans B., Bernoth E.M., Bromage N., Bunyan J., Erhartd W., Flecknell P., Gregory N., Hackbarth H.: Recommendation for euthanasia of experimental animals: part 1. Laboratory animals 1996, 30, 293-316.

9. Del Alamo A.M., Mandsager R.E., Riebold T.W., Payton M.E.: Evaluation of intravenous administration of alfoxalone, propofol, and ketamine-diazepam for anesthesia in alpacas. Vet Anaesth Analgesia 2015, 42, 72-82.

10. Driessen B.: Assisted recovery in horses awakening from general anesthesia. Int Vet Inform Service, Ithaca NY (www.ivis.org)

11. Dugdale A.: Veterinary anaesthesia principles to practice. Blackwell Publishing Ltd, UK, 2010.

12. Erdine S., Yücel A., Ozyalçin S., Ozyuvacı E., Talu G.K., Ahiskalı B., Apak H., Savci N.: Neurotoxicity of midazolam in the rabbit. Pain 1999, 80, 419-423.

13. Fredriksson A., Ponten E., Gordh T., Eriksson P.: Neonatal exposure to a combination of N-Methyl-D aspartate and aminobutyric acid type a receptor anesthetic agents potentiates apoptotic neurodegeneration and persistent behavioral deficits. Anesthesiology 2007, 107, 427-436.

14. Gianotti G., Valverde A., Sinclair M., Dyson D.H., Gibson T., Johnson R.: Prior determination of baseline minimum alveolar concentration (MAC) of isoflurane does not influence the effect of ketamine on MAC in rabbits. C J Vet Res 2012, 76, 261-267.

15. Greene A.: Veterinary anesthesia and pain management secrets, Hanley \& Belfus Inc., Philadelphia, 2002.

16. Grint N.J., Murison P.J.: A comparison of ketamine-midazolam and ketamine-medetomidine combinations for induction of anaesthesia in rabbits. Vet Anaesth Analgesia 2007, 35, 113-121.

17. Grint N.J., Murison P.J.: Peri-operative body temperatures in isoflurane-anaesthetized rabbits following ketamine-midazolam or ketamine-medetomidine. Vet Anaesth Analgesia 2008, 34, 181-189.

18. Hall L.W., Clarke K.W., Trim C.M.: Veterinary Anaesthesia, W.B. Saunders, Philadelphia 2000

19. Henke J., Astner S., Brill T., Eissner B., Busch R., Erhardt W.: Comparative study of three intramuscular anaesthetic combinations (medetoidine/ketamine, medetomidine /fentanyl/ midazolam and xylazine/ketamine) in rabbits. Vet Anaesth Analgesia 2005, 32, 261-270.

20. Lee H.J., Kim J.W., Yim S.V., Kim M.J., Kim S.A., Kim Y.J., Kim C.J. Chung J.H.: Fluoxetine enhances cell proliferation and prevents apoptosis in dentate gyrus of maternally separated rats. Mol Psychiatry 2001, 610, 725-728.

21. Loftis G.K., Collins S., McDowell M.: Anesthesia-induced neuronal apoptosis during synaptogenesis: A review of the literature. Am J Nurse Anesth 2012, 80, 291-298.

22. Massoco C., Palermo-Neto J.: Effects of midazolam on equine innate immune response: a flow cytometric study. Vet Immunol Immunopathol 2003, 95, 11-19.

23. Orszulak-Michalak D., Owczarek J., Wiktorowska-Owczarek A.K.: Influence of midazolam pharmocokinetics of verapamil in rabbits. Pol J Pharmacol 2002, 54, 501-506.

24. Ozkan C., Kaya A., Akgül Y.: Normal values of hematological and some biochemical parameters in serum and urine of New Zealand white rabbits. World Rabbit Sci 2012, 20, 253-259.

25. Schroeder A.C., Smith L.J.: Respiratory rates and arterial bloodgas tensions in healthy rabbits given buprenorphine, butorphanol, midazolam, or their combinations. J Am Assoc Lab Anim Sci, 2011, 50, 205-211.

26. Stevens M.F., Werdehausen R., Gaza N., Hermanns H., Kremer D., Bauer I., Küry P., Hollmann M.W., Braun S.: Midazolam activates the intrinsic pathway of apoptosis independent of benzodiazepine and death receptor signaling. Reg Anesth Pain Med 2011, 36, 343-349.

27. Wells S.M., Sleeper M.: Cardiac troponins. J Vet Emerg Critical Care, 2008, 18, 235-245.

28. Yamashita K., Tsubakishita S., Futaoka S., Ueda I., Hamaguci H., Seno T., Katoh S., Uzimasawa Y., Muir W.W.: Cardiovascular effects of medetomidine, detomidine and xylazine in horses. J Vet Med Sci 2000, 62, 1025-1032. 